\title{
Differential sandwich theorems of $p$-valent analytic functions involving a linear operator
}

\author{
M. K. Aouf and T. M. Seoudy
}

\begin{abstract}
In this paper we derive some subordination and superordination results for certain $p$-valent analytic functions in the open unit disc, which are acted upon by a class of a linear operator. Some of our results improve and generalize previously known results.
\end{abstract}

\section{Introduction}

Let $H(U)$ denotes the class of analytic functions in the open unit disc $U=$ $\{z \in \mathbb{C}:|z|<1\}$ and let $H[a, p]$ denotes the subclass of the functions $f \in H(U)$ of the form:

$$
f(z)=a+a_{p} z^{p}+a_{p+1} z^{p+1}+\ldots(a \in \mathbb{C} ; p \in \mathbb{N}=\{1,2, . .\}) .
$$

Also, let $\mathcal{A}(p)$ be the subclass of the functions $f \in H(U)$ of the form:

$$
f(z)=z^{p}+\sum_{k=p+1}^{\infty} a_{k} z^{k} \quad(p \in \mathbb{N}),
$$

and set $\mathcal{A} \equiv \mathcal{A}(1)$. For functions $f(z) \in A(p)$, given by (1.1), and $g(z)$ given by

$$
g(z)=z^{p}+\sum_{k=p+1}^{\infty} b_{k} z^{k} \quad(p \in \mathbb{N}),
$$

Key Words: Analytic function, Hadamard product, differential subordination, superordination, linear operator.

2010 Mathematics Subject Classification: 30C45.

Received: April, 2011.

Revised: April, 2011.

Accepted: February, 2012. 
the Hadamard product (or convolution) of $f(z)$ and $g(z)$ is defined by

$$
(f * g)(z)=z^{p}+\sum_{k=p+1}^{\infty} a_{k} b_{k} z^{k}=(g * f)(z) .
$$

For $f, g \in H(U)$, we say that the function $f$ is subordinate to $g$, if there exists a Schwarz function $w$, i.e, $w \in H(U)$ with $w(0)=0$ and $|w(z)|<1$, $z \in U$, such that $f(z)=g(w(z))$ for all $z \in U$. This subordination is usually denoted by $f(z) \prec g(z)$. It is well-known that, if the function $g$ is univalent in $U$, then $f(z) \prec g(z)$ is equivalent to $f(0)=g(0)$ and $f(U) \subset g(U)$ (see [5] and $[9])$.

Supposing that $h$ and $k$ are two analytic functions in $U$, let

$$
\phi(r, s, t ; z): \mathbb{C}^{3} \times U \rightarrow \mathbb{C} .
$$

If $h$ and $\varphi\left(h(z), z h^{\prime}(z), z^{2} h^{\prime \prime}(z) ; z\right)$ are univalent functions in $U$ and if $h$ satisfies the second-order superordination

$$
k(z) \prec \varphi\left(h(z), z h^{\prime}(z), z^{2} h^{\prime \prime}(z) ; z\right),
$$

then $h$ is called to be a solution of the differential superordination (1.4). A function $q \in H(U)$ is called a subordinant of (1.4), if $q(z) \prec h(z)$ for all the functions $h$ satisfying (1.4). A univalent subordinant $\widetilde{q}$ that satisfies $q(z) \prec$ $\widetilde{q}(z)$ for all of the subordinants $q$ of (1.4), is said to be the best subordinant.

Recently, Miller and Mocanu [10] obtained sufficient conditions on the functions $k, q$ and $\varphi$ for which the following implication holds:

$$
k(z) \prec \varphi\left(h(z), z h^{\prime}(z), z^{2} h^{\prime \prime}(z) ; z\right) \Rightarrow q(z) \prec h(z) .
$$

Using these results, Bulboaca [3] considered certain classes of first-order differential superordinations, as well as superordination-preserving integral operators [4]. Ali et al. [1], using the results from [3], obtained sufficient conditions for certain normalized analytic functions to satisfy

$$
q_{1}(z) \prec \frac{z f^{\prime}(z)}{f(z)} \prec q_{2}(z),
$$

where $q_{1}$ and $q_{2}$ are given univalent normalized functions in $U$.

For complex parameters

$$
\alpha_{1}, \ldots, \alpha_{q} \text { and } \beta_{1}, \ldots, \beta_{s}\left(\beta_{j} \notin \mathbb{Z}_{0}^{-}=\{0,-1,-2, \ldots\} ; j=1,2, \ldots, s\right),
$$

we now define the generalized hypergeometric function ${ }_{q} F_{s}\left(\alpha_{1}, \ldots, \alpha_{q} ; \beta_{1}, \ldots, \beta_{s} ; z\right)$ by (see, for example, [15, p.19])

$$
{ }_{q} F_{s}\left(\alpha_{1}, \ldots, \alpha_{q} ; \beta_{1}, \ldots, \beta_{s} ; z\right)=\sum_{k=0}^{\infty} \frac{\left(\alpha_{1}\right)_{k} \ldots\left(\alpha_{q}\right)_{k}}{\left(\beta_{1}\right)_{k} \ldots\left(\beta_{s}\right)_{k}} \cdot \frac{z^{k}}{k !}
$$




$$
\left(q \leq s+1 ; q, s \in \mathbb{N}_{0}=\mathbb{N} \cup\{0\} ; z \in U\right),
$$

where $(\theta)_{\nu}$ is the Pochhammer symbol defined, in terms of the Gamma function $\Gamma$, by

$$
(\theta)_{\nu}=\frac{\Gamma(\theta+\nu)}{\Gamma(\theta)}=\left\{\begin{array}{rr}
1 & \left(\nu=0 ; \theta \in \mathbb{C}^{*}=\mathbb{C} \backslash\{0\}\right) \\
\theta(\theta+1) \ldots(\theta+\nu-1) \quad(\nu \in \mathbb{N} ; \theta \in \mathbb{C})
\end{array}\right.
$$

Let

$$
\begin{aligned}
h\left(\alpha_{1}, \ldots, \alpha_{q} ; \beta_{1}, \ldots, \beta_{s} ; z\right) & =z^{p}{ }_{q} F_{s}\left(\alpha_{1}, \ldots, \alpha_{q} ; \beta_{1}, \ldots, \beta_{s} ; z\right) \\
& =z^{p}+\sum_{k=p+1}^{\infty} \Gamma_{p, q, s}\left(\alpha_{1}\right) z^{k},
\end{aligned}
$$

where

$$
\Gamma_{p, q, s}\left(\alpha_{1}\right)=\frac{\left(\alpha_{1}\right)_{k-p} \ldots\left(\alpha_{q}\right)_{k-p}}{\left(\beta_{1}\right)_{k-p} \ldots\left(\beta_{s}\right)_{k-p}(1)_{k-p}},
$$

and using the Hadamard product, El-Ashwah and Aouf [7] defined the following operator

$$
I_{p, \lambda}^{m, \ell}\left(\alpha_{1}, \ldots, \alpha_{q} ; \beta_{1}, \ldots, \beta_{s}\right): A(p) \rightarrow A(p)
$$

by

$$
\begin{gathered}
I_{p, \lambda}^{0, \ell}\left(\alpha_{1}, \ldots, \alpha_{q} ; \beta_{1}, \ldots, \beta_{s}\right) f(z)=f(z) * h\left(\alpha_{1}, \ldots, \alpha_{q} ; \beta_{1}, \ldots, \beta_{s} ; z\right) ; \\
I_{p, \lambda}^{1, \ell}\left(\alpha_{1}, \ldots, \alpha_{q} ; \beta_{1}, \ldots, \beta_{s}\right) f(z)=(1-\lambda)\left(f(z) * h\left(\alpha_{1}, \ldots, \alpha_{q} ; \beta_{1}, \ldots, \beta_{s} ; z\right)\right) \\
+\frac{\lambda}{(p+\ell) z^{\ell-1}}\left(z^{\ell} f(z) * h\left(\alpha_{1}, \ldots, \alpha_{q} ; \beta_{1}, \ldots, \beta_{s} ; z\right)\right)^{\prime} ;
\end{gathered}
$$

and

$$
I_{p, \lambda}^{m, \ell}\left(\alpha_{1}, \ldots, \alpha_{q} ; \beta_{1}, \ldots, \beta_{s}\right) f(z)=I_{p, q, s, \lambda}^{1, \ell}\left(I_{p, q, s, \lambda}^{m-1, \ell}\left(\alpha_{1}, \ldots, \alpha_{q} ; \beta_{1}, \ldots, \beta_{s}\right) f(z)\right) .
$$

If $f \in A(p)$, then from (1.1) and (1.8), we can easily see that

$$
\begin{gathered}
I_{p, \lambda}^{m, \ell}\left(\alpha_{1}, \ldots, \alpha_{q} ; \beta_{1}, \ldots, \beta_{s}\right) f(z)=z^{p}+\sum_{k=p+1}^{\infty}\left[\frac{p+\ell+\lambda(k-p)}{p+\ell}\right]^{m} \Gamma_{p, q, s}\left(\alpha_{1}\right) a_{k} z^{k} \\
\left(p \in \mathbb{N} ; m \in \mathbb{N}_{0}=\mathbb{N} \cup\{0\} ; \ell \geq 0 ; \lambda \geq 0 ; z \in U\right)
\end{gathered}
$$

It can be easily verified from the definition (1.9) that:

$$
z\left(I_{p, q, s, \lambda}^{m, \ell}\left(\alpha_{1}\right) f(z)\right)^{\prime}=\alpha_{1} I_{p, q, s, \lambda}^{m, \ell}\left(\alpha_{1}+1\right) f(z)-\left(\alpha_{1}-p\right) I_{p, q, s, \lambda}^{m, \ell}\left(\alpha_{1}\right) f(z),
$$


where

$$
I_{p, q, s, \lambda}^{m, \ell}\left(\alpha_{1}\right) f(z)=I_{p, \lambda}^{m, \ell}\left(\alpha_{1}, \ldots, \alpha_{q} ; \beta_{1}, \ldots, \beta_{s}\right) f(z) .
$$

It should be remarked that the linear operator $I_{p, q, s, \lambda}^{m, \ell}\left(\alpha_{1}\right)$ is a generalization of many other linear operators considered earlier. In particular, we have

$$
I_{p, q, s, \lambda}^{0, \ell}\left(\alpha_{1}\right) f(z)=H_{p, q, s}\left(\alpha_{1}\right) f(z),
$$

where the linear operator $H_{p, q, s}\left(\alpha_{1}\right)$ was investigated by Dziok and Srivastava [8], and also we have

$$
I_{p, 2,1, \lambda}^{0, \ell}(a, 1 ; c) f(z)=L_{p}(a, c) f(z)\left(a \in \mathbb{R} ; c \in \mathbb{R} \backslash \mathbb{Z}_{0}^{-}\right),
$$

where the linear operator $L_{p}(a, c)$ was studied by Saitoh [13] which yields the operator $L(a, c) f(z)$ introduced by Carlson and Shaffer [6] for $p=1$.

\section{Preliminaries}

In order to prove our subordination and superordination results, we make use of the following known definition and results.

Definition [10]. Denote by $Q$ the set of all functions $f(z)$ that are analytic and injective on $\bar{U} \backslash E(f)$, where

$$
E(f)=\left\{\zeta: \zeta \in \partial \text { and } \lim _{z \rightarrow \zeta} f(z)=\infty\right\}
$$

and are such that $f^{\prime}(\zeta) \neq 0$ for $\zeta \in \partial U \backslash E(f)$.

Lemma 1 [9]. Let the function $q(z)$ be univalent in the unit disc $U$ and let $\theta$ and $\varphi$ be analytic in a domain $D$ containing $q(U)$ with $\varphi(w) \neq 0$ when $w \in q(U)$. Set $Q(z)=z q^{\prime}(z) \varphi(q(z))$ and $h(z)=\theta(q(z))+Q(z)$. Suppose that

(i) $Q(z)$ is starlike univalent in $U$,

(ii) $\Re\left(\frac{z h^{\prime}(z)}{Q(z)}\right)>0$ for $z \in U$.

If $p$ is analytic with $p(0)=q(0), p(U) \subseteq D$ and

$$
\theta(p(z))+z p^{\prime}(z) \varphi(p(z)) \prec \theta(q(z))+z q^{\prime}(z) \varphi(q(z))
$$

then $p(z) \prec q(z)$ and $q(z)$ is the best dominant.

Lemma 2 [5]. Let $q(z)$ be convex univalent in the unit disc $U$ and let $\theta$ and $\varphi$ be analytic in a domain $D$ containing $q(U)$. Suppose that

(i) $\Re\left\{\frac{\theta^{\prime}(q(z))}{\varphi(q(z))}\right\}>0$ for $z \in U$; 
(ii) $z q^{\prime}(z) \varphi(q(z))$ is starlike univalent in $U$.

If $p(z) \in H[q(0), 1] \cap Q$, with $p(U) \subseteq D$, and $\theta(p(z))+z p^{\prime}(z) \varphi(p(z))$ is univalent in $U$, and

$$
\theta(q(z))+z q^{\prime}(z) \varphi(q(z)) \prec \theta(p(z))+z p^{\prime}(z) \varphi(p(z)),
$$

then $q(z) \prec p(z)$ and $q(z)$ is the best subordinant

The following lemma gives us a necessary and sufficient condition for the univalence of a special function which will be used in some particular case.

Lemma 3 [12]. The function $q(z)=(1-z)^{-2 a b}\left(a, b \in \mathbb{C}^{*}\right)$ is univalent in the unit disc $U$ if and only if $|2 a b-1| \leq 1$ or $|2 a b+1| \leq 1$.

\section{Main Results}

Unless otherwise mentioned, we assume throughout this paper that $p \in \mathbb{N}, m \in$ $\mathbb{N}_{0}, \ell \geq 0 ; \lambda \geq 0$ and the power understood as principal values.

Theorem 1. Let $q(z)$ be univalent in $U$ such that $q(0)=1, q(z) \neq 0$ and $\frac{z q^{\prime}(z)}{q(z)}$ is starlike in $U$. Let $f \in \mathcal{A}(p)$ and suppose that $f$ and $q$ satisfy the next conditions:

$$
\left[\frac{I_{p, q, s, \lambda}^{m, \ell}\left(\alpha_{1}\right) f(z)}{z^{p}}\right]^{\mu}\left[\frac{z^{p}}{I_{p, q, s, \lambda}^{m, \ell}\left(\alpha_{1}+1\right) f(z)}\right]^{\eta} \neq 0\left(\mu \in \mathbb{C}^{*} ; \eta \in \mathbb{C} ; z \in U\right),
$$

and

$\Re\left\{1+\frac{\zeta}{\gamma} q(z)+\frac{2 \delta}{\gamma}[q(z)]^{2}-\frac{z q^{\prime}(z)}{q(z)}+\frac{z q^{\prime \prime}(z)}{q^{\prime}(z)}\right\}>0 \quad\left(\zeta, \delta \in \mathbb{C} ; \gamma \in \mathbb{C}^{*} ; z \in U\right)$.

If

$$
\Psi(z) \prec \chi+\zeta q(z)+\delta[q(z)]^{2}+\gamma \frac{z q^{\prime}(z)}{q(z)},
$$

where

$$
\begin{aligned}
\Psi(z)= & \chi+\zeta\left[\frac{I_{p, q, s, \lambda}^{m, \ell}\left(\alpha_{1}\right) f(z)}{z^{p}}\right]^{\mu}\left[\frac{z^{p}}{I_{p, q, s, \lambda}^{m, \ell}\left(\alpha_{1}+1\right) f(z)}\right]^{\eta} \\
& +\delta\left[\frac{I_{p, q, s, \lambda}^{m, \ell}\left(\alpha_{1}\right) f(z)}{z^{p}}\right]^{2 \mu}\left[\frac{z^{p}}{I_{p, q, s, \lambda}^{m, \ell}\left(\alpha_{1}+1\right) f(z)}\right]^{2 \eta} \\
& +\gamma \mu \alpha_{1}\left[\frac{I_{p, q, s, \lambda}^{m, \ell}\left(\alpha_{1}+1\right) f(z)}{I_{p, q, s, \lambda}^{m, \ell}\left(\alpha_{1}\right) f(z)}-1\right]
\end{aligned}
$$




$$
+\gamma \eta\left(\alpha_{1}+1\right)\left[1-\frac{I_{p, q, s, \lambda}^{m, \ell}\left(\alpha_{1}+2\right) f(z)}{I_{p, q, s, \lambda}^{m, \ell}\left(\alpha_{1}+1\right) f(z)}\right]
$$

then

$$
\left[\frac{I_{p, q, s, \lambda}^{m, \ell}\left(\alpha_{1}\right) f(z)}{z^{p}}\right]^{\mu}\left[\frac{z^{p}}{I_{p, q, s, \lambda}^{m, \ell}\left(\alpha_{1}+1\right) f(z)}\right]^{\eta} \prec q(z),
$$

and $q$ is the best dominant of (3.3).

Proof. Let

$$
h(z)=\left[\frac{I_{p, q, s, \lambda}^{m, \ell}\left(\alpha_{1}\right) f(z)}{z^{p}}\right]^{\mu}\left[\frac{z^{p}}{I_{p, q, s, \lambda}^{m, \ell}\left(\alpha_{1}+1\right) f(z)}\right]^{\eta}(z \in U) .
$$

According to (3.1) the function $h(z)$ is analytic in $U$, and differentiating (3.5) logarithmically with respect to $z$, we obtain

$$
\frac{z h^{\prime}(z)}{h(z)}=\mu\left[\frac{z\left(I_{p, q, s, \lambda}^{m, \ell}\left(\alpha_{1}\right) f(z)\right)^{\prime}}{I_{p, q, s, \lambda}^{m, \ell}\left(\alpha_{1}\right) f(z)}-p\right]+\eta\left[p-\frac{z\left(I_{p, q, s, \lambda}^{m, \ell}\left(\alpha_{1}+1\right) f(z)\right)^{\prime}}{I_{p, q, s, \lambda}^{m, \ell}\left(\alpha_{1}+1\right) f(z)}\right] .
$$

By using the identity (1.10), we obtain

$$
\frac{z h^{\prime}(z)}{h(z)}=\mu \alpha_{1}\left[\frac{I_{p, q, s, \lambda}^{m, \ell}\left(\alpha_{1}+1\right) f(z)}{I_{p, q, s, \lambda}^{m, \ell}\left(\alpha_{1}\right) f(z)}-1\right]+\eta\left(\alpha_{1}+1\right)\left[1-\frac{I_{p, q, s, \lambda}^{m, \ell}\left(\alpha_{1}+2\right) f(z)}{I_{p, q, s, \lambda}^{m, \ell}\left(\alpha_{1}+1\right) f(z)}\right] .
$$

In order to prove our result we will use Lemma 1. In this lemma consider

$$
\theta(w)=\chi+\zeta w+\delta w^{2} \quad \text { and } \quad \varphi(w)=\frac{\gamma}{w}
$$

then $\theta$ is analytic in $\mathbb{C}$ and $\varphi(w) \neq 0$ is analytic in $\mathbb{C}^{*}$.Also, if we let

$$
Q(z)=z q^{\prime}(z) \varphi(q(z))=\gamma \frac{z q^{\prime}(z)}{q(z)}
$$

and

$$
g(z)=\theta(q(z))+Q(z)=\chi+\zeta q(z)+\delta[q(z)]^{2}+\gamma \frac{z q^{\prime}(z)}{q(z)} .
$$

We see that $Q(z)$ is starlike function in $U$. From (3.2), we also have

$$
\Re\left\{\frac{z g^{\prime}(z)}{Q(z)}\right\}=\Re\left\{1+\frac{\zeta}{\gamma} q(z)+\frac{2 \delta}{\gamma}[q(z)]^{2}-\frac{z q^{\prime}(z)}{q(z)}+\frac{z q^{\prime \prime}(z)}{q^{\prime}(z)}\right\}>0 \quad(z \in U),
$$


and then, by using Lemma 1 we deduce that the subordination (3.3) implies $h(z) \prec q(z)$, and the function $q$ is the best dominant of (3.3).

Putting $q=2, s=p=1, m=0, \alpha_{1}=a(a \in \mathbb{C}), \alpha_{2}=1$ and $\beta_{1}=c$ $\left(c \in \mathbb{C} \backslash \mathbb{Z}_{0}^{-}\right)$in Theorem 1 , we obtain the following result which improves the corresponding work of Shammugam et al. [14, Theorem 3.1].

Corollary 1. Let $q(z)$ be univalent in $U$ such that $q(0)=1, q(z) \neq 0$ and $\frac{z q^{\prime}(z)}{q(z)}$ is starlike in $U$. Let $f \in \mathcal{A}$ such that

$$
\left[\frac{L(a, c) f(z)}{z}\right]^{\mu}\left[\frac{z}{L(a+1, c) f(z)}\right]^{\eta} \neq 0 \quad\left(\mu \in \mathbb{C}^{*} ; z \in U\right)
$$

and suppose that $q$ satisfies (3.2).If

$$
\Lambda(z) \prec \chi+\zeta q(z)+\delta[q(z)]^{2}+\gamma \frac{z q^{\prime}(z)}{q(z)}
$$

where

$$
\begin{aligned}
\Lambda(z)= & \chi+\zeta\left[\frac{L(a, c) f(z)}{z}\right]^{\mu}\left[\frac{z}{L(a+1, c) f(z)}\right]^{\eta} \\
& +\delta\left[\frac{L(a, c) f(z)}{z}\right]^{2 \mu}\left[\frac{z}{L(a+1, c) f(z)}\right]^{2 \eta} \\
& +\gamma \mu a\left[\frac{L(a+1, c) f(z)}{L(a, c) f(z)}-1\right] \\
+ & \gamma \eta(a+1)\left[1-\frac{L(a+2, c) f(z)}{L(a+1, c) f(z)}\right]
\end{aligned}
$$

then

$$
\left[\frac{L(a, c) f(z)}{z}\right]^{\mu}\left[\frac{z}{L(a+1, c) f(z)}\right]^{\eta} \prec q(z),
$$

and $q$ is the best dominant of (3.7).

Putting $q(z)=\frac{1+A z}{1+B z}(-1 \leq B<A \leq 1)$ in Corollary 1 , we obtain the following result which improves the corresponding work of Shammugam et al. [14, Corollary 3.2].

Corollary 2. Assume that

$$
\begin{gathered}
\Re\left\{\frac{1-A B z^{2}}{(1+A z)(1+B z)}+\frac{\zeta}{\gamma}\left[\frac{1+A z}{1+B z}\right]+\frac{2 \delta}{\gamma}\left[\frac{1+A z}{1+B z}\right]^{2}\right\}>0 \\
\left(\zeta, \delta \in \mathbb{C} ; \gamma \in \mathbb{C}^{*} ; z \in U\right)
\end{gathered}
$$


holds. Let $f \in \mathcal{A}$ such that (3.6) holds. If

$$
\Lambda(z) \prec \chi+\zeta \frac{1+A z}{1+B z}+\delta\left[\frac{1+A z}{1+B z}\right]^{2}+\frac{\gamma(A-B) z}{(1+A z)(1+B z)},
$$

where $\Lambda(z)$ is given by (3.8), then

$$
\left[\frac{L(a, c) f(z)}{z}\right]^{\mu}\left[\frac{z}{L(a+1, c) f(z)}\right]^{\eta} \prec \frac{1+A z}{1+B z}
$$

and $\frac{1+A z}{1+B z}$ is the best dominant of (3.9).

Putting $q(z)=\left(\frac{1+z}{1-z}\right)^{\vartheta}(0<\vartheta \leq 1)$ in Corollary 1, we obtain the following result which improves the corresponding work of Shammugam et al. [14, Corollary 3.3].

Corollary 3. Assume that

$$
\Re\left\{\frac{1-3 z^{2}}{1-z^{2}}+\frac{\zeta}{\gamma}\left[\frac{1+z}{1-z}\right]^{\vartheta}+\frac{2 \delta}{\gamma}\left[\frac{1+z}{1-z}\right]^{2 \vartheta}\right\}>0 \quad\left(\zeta, \delta \in \mathbb{C} ; \gamma \in \mathbb{C}^{*} ; z \in U\right)
$$

holds. Let $f \in \mathcal{A}$ such that (3.6) holds. If

$$
\Lambda(z) \prec \chi+\zeta\left(\frac{1+z}{1-z}\right)^{\vartheta}+\delta\left(\frac{1+z}{1-z}\right)^{2 \vartheta}+\frac{2 \gamma \vartheta z}{\left(1-z^{2}\right)}(0<\vartheta \leq 1),
$$

where $\Lambda(z)$ is given by (3.8), then

$$
\left[\frac{L(a, c) f(z)}{z}\right]^{\mu}\left[\frac{z}{L(a+1, c) f(z)}\right]^{\eta} \prec\left(\frac{1+z}{1-z}\right)^{\vartheta}
$$

and $\left(\frac{1+z}{1-z}\right)^{\vartheta}$ is the best dominant of (3.10).

Putting $q(z)=e^{\mu A z}(|\mu A|<\pi)$ in Corollary 1, we obtain the following result which improves the corresponding work of Shammugam et al. [14, Corollary 3.4].

Corollary 4. Assume that

$$
\Re\left\{1+\frac{\zeta}{\gamma} e^{\mu A z} q(z)+\frac{2 \delta}{\gamma} e^{2 \mu A z}\right\}>0 \quad\left(\zeta, \delta \in \mathbb{C} ; \gamma \in \mathbb{C}^{*} ; z \in U\right)
$$

holds and let $f \in \mathcal{A}$ such that (3.6) holds. If

$$
\Lambda(z) \prec \chi+\zeta e^{\mu A z}+\delta e^{2 \mu A z}+\gamma A \mu z \quad(|\mu A|<\pi),
$$


where $\Lambda(z)$ is given by (3.8), then

$$
\left[\frac{L(a, c) f(z)}{z}\right]^{\mu}\left[\frac{z}{L(a+1, c) f(z)}\right]^{\eta} \prec e^{\mu A z}
$$

and $e^{\mu A z}$ is the best dominant of (3.11).

Putting $q=s+1, \alpha_{i}=1(i=1, . ., s+1), \beta_{j}=1(j=1, . ., s), m=\zeta=$ $\delta=0, \chi=p=1, \gamma=\frac{1}{a b}\left(a, b \in \mathbb{C}^{*}\right), \mu=a, \eta=0$ and $q(z)=(1-z)^{-2 a b}$ in Theorem 1, then combining this to gather with Lemma 3 we obtain the next result due to Obradovic et al. [11, Theorem 1].

Corollary 5 [11]. Let $a, b \in \mathbb{C}^{*}$ such that $|2 a b-1| \leq 1$ or $|2 a b+1| \leq 1$. Let $f \in \mathcal{A}$ and suppose that $\frac{f(z)}{z} \neq 0$ for all $z \in U$. If

$$
1+\frac{1}{b}\left(\frac{z f^{\prime}(z)}{f(z)}-1\right) \prec \frac{1+z}{1-z},
$$

then

$$
\left(\frac{f(z)}{z}\right)^{a} \prec(1-z)^{-2 a b}
$$

and $(1-z)^{-2 a b}$ is the best dominant of (3.12).

Remark 1. For $a=1$, Corollary 5 reduces to the recent result of Srivastava and Lashin [16].

Putting $q=s+1, \alpha_{i}=1(i=1, . ., s+1), \beta_{j}=1(j=1, . ., s), m=\zeta=\delta=$ $0, \chi=p=\gamma=1, \eta=0$ and $q(z)=(1+B z)^{\frac{\mu(A-B)}{B}}$ in Theorem 2, and using Lemma 2 we obtain the next result.

Corollary 6. Let $-1 \leq A<B \leq 1$ with $B \neq 0$, and suppose that $\left|\frac{\mu(A-B)}{B}-1\right| \leq$ 1 or $\left|\frac{\mu(A-B)}{B}+1\right| \leq 1$. Let $f \in \mathcal{A}$ such that $\frac{f(z)}{z} \neq 0$ for all $z \in U$, and let $\mu \in \mathbb{C}^{*}$. If

$$
1+\mu\left(\frac{z f^{\prime}(z)}{f(z)}-1\right) \prec \frac{1+[B+\mu(A-B)] z}{1+B z}
$$

then

$$
\left(\frac{f(z)}{z}\right)^{\mu} \prec(1+B z)^{\frac{\mu(A-B)}{B}}
$$

and $(1+B z)^{\frac{\mu(A-B)}{B}}$ is the best dominant of (3.13).

Putting $q=s+1, \alpha_{i}=1(i=1, . ., s+1), \beta_{j}=1(j=1, . ., s), m=\zeta=$ $\delta=0, \chi=p=1, \gamma=\frac{e^{i \tau}}{a b \cos \tau}\left(a, b \in \mathbb{C}^{*} ;|\tau|<\frac{\pi}{2}\right), \mu=a, \eta=0$ and $q(z)=$ $(1-z)^{-2 a b \cos \tau e^{-i \tau}}$ in Theorem 1 , we obtain the following result due to Aouf et al. [2, Theorem 1]. 
Corollary 7 [2]. Let $a, b \in \mathbb{C}^{*},|\tau|<\frac{\pi}{2}$ and suppose that $\left|2 a b \cos \tau e^{-i \tau}-1\right| \leq$ 1 or $\left|2 a b \cos \tau e^{-i \tau}+1\right| \leq 1$. Let $f \in \mathcal{A}$ and suppose that $\frac{f(z)}{z} \neq 0$ for all $z \in U$. If

$$
1+\frac{e^{i \tau}}{b \cos \tau}\left(\frac{z f^{\prime}(z)}{f(z)}-1\right) \prec \frac{1+z}{1-z}
$$

then

$$
\left(\frac{f(z)}{z}\right)^{a} \prec(1-z)^{-2 a b \cos \tau e^{-i \tau}}
$$

and $(1-z)^{-2 a b \cos \tau e^{-i \tau}}$ is the best dominant of (3.14).

Theorem 2. Let $q$ be convex in $U$ such that $q(0)=1$ and $\frac{z q^{\prime}(z)}{q(z)}$ is starlike in $U$. Further assume that

$$
\Re\left\{(\zeta+2 \delta q(z)) \frac{q(z) q^{\prime}(z)}{\gamma}\right\}>0 \quad\left(\zeta, \delta \in \mathbb{C} ; \gamma \in \mathbb{C}^{*}\right) .
$$

Let $f \in \mathcal{A}(p)$ such that

$$
0 \neq\left[\frac{I_{p, q, s, \lambda}^{m, \ell}\left(\alpha_{1}\right) f(z)}{z^{p}}\right]^{\mu}\left[\frac{z^{p}}{I_{p, q, s, \lambda}^{m, \ell}\left(\alpha_{1}+1\right) f(z)}\right]^{\eta} \in H[q(0), 1] \cap Q .
$$

If $\Psi(z)$ given by (3.4) is univalent in $U$ and satisfies the following superordination condition

$$
\chi+\zeta q(z)+\delta[q(z)]^{2}+\gamma \frac{z q^{\prime}(z)}{q(z)} \prec \Psi(z)
$$

then

$$
q(z) \prec\left[\frac{I_{p, q, s, \lambda}^{m, \ell}\left(\alpha_{1}\right) f(z)}{z^{p}}\right]^{\mu}\left[\frac{z^{p}}{I_{p, q, s, \lambda}^{m, \ell}\left(\alpha_{1}+1\right) f(z)}\right]^{\eta},
$$

and $q$ is the best subordinant of (3.17).

Putting $q=2, s=p=1, m=0, \alpha_{1}=a(a \in \mathbb{C}), \alpha_{2}=1$ and $\beta_{1}=c$ $\left(c \in \mathbb{C} \backslash \mathbb{Z}_{0}^{-}\right)$in Theorem 2 , we obtain the following result which improves the corresponding work of Shammugam et al. [14, Theorem 3.11].

Corollary 8. Let $q$ be convex in $U$ such that $q(0)=1$ and $\frac{z q^{\prime}(z)}{q(z)}$ is starlike in $U$. Further assume that (3.15) holds. Let $f \in \mathcal{A}$ such that

$$
0 \neq\left[\frac{L(a, c) f(z)}{z}\right]^{\mu}\left[\frac{z}{L(a+1, c) f(z)}\right]^{\eta} \in H[q(0), 1] \cap Q .
$$


If $\Lambda(z)$ given by (3.8) is univalent in $U$ and satisfies the following superordination condition

$$
\chi+\zeta q(z)+\delta[q(z)]^{2}+\gamma \frac{z q^{\prime}(z)}{q(z)} \prec \Lambda(z)
$$

then

$$
q(z) \prec\left[\frac{L(a, c) f(z)}{z}\right]^{\mu}\left[\frac{z}{L(a+1, c) f(z)}\right]^{\eta}
$$

and $q$ is the best subordinant of (3.19).

Combining Theorems 1 and 2, we obtain the following two sandwich results: Theorem 3. Let $q_{i}$ be two convex functions in $U$ such that $q_{i}(0)=1$ and $\frac{z q_{i}^{\prime}(z)}{q_{i}(z)}(i=1,2)$ is starlike in $U$. Suppose that $q_{1}(z)$ satisfies $(3.15)$ and $q_{2}(z)$ satisfies (3.2). Let $f \in \mathcal{A}(p)$ and suppose that

$$
\left[\frac{I_{p, q, s, \lambda}^{m, \ell}\left(\alpha_{1}\right) f(z)}{z^{p}}\right]^{\mu}\left[\frac{z^{p}}{I_{p, q, s, \lambda}^{m, \ell}\left(\alpha_{1}+1\right) f(z)}\right]^{\eta} \in H[q(0), 1] \cap Q .
$$

If $\Psi(z)$ given by (3.4) is univalent in $U$, and

$\chi+\zeta q_{1}(z)+\delta\left[q_{1}(z)\right]^{2}+\gamma \frac{z q_{1}^{\prime}(z)}{q_{1}(z)} \prec \Psi(z) \prec \chi+\zeta q_{2}(z)+\delta\left[q_{2}(z)\right]^{2}+\gamma \frac{z q_{2}^{\prime}(z)}{q_{2}(z)}$,

then

$$
q_{1}(z) \prec\left[\frac{I_{p, q, s, \lambda}^{m, \ell}\left(\alpha_{1}\right) f(z)}{z^{p}}\right]^{\mu}\left[\frac{z^{p}}{I_{p, q, s, \lambda}^{m, \ell}\left(\alpha_{1}+1\right) f(z)}\right]^{\eta} \prec q_{2}(z),
$$

and $q_{1}$ and $q_{2}$ are, respectively, the best subordinant and the best dominant of $(3.20)$.

Putting $q=2, s=p=1, m=0, \alpha_{1}=a(a \in \mathbb{C}), \alpha_{2}=1$ and $\beta_{1}=c$ $\left(c \in \mathbb{C} \backslash \mathbb{Z}_{0}^{-}\right)$in Theorem 3, we obtain the following result which improves the corresponding work of Shammugam et al. [14, Theorem 3.12].

Corollary 9. Let $q_{i}$ be two convex functions in $U$ such that $q_{i}(0)=1$ and $\frac{z q_{i}^{\prime}(z)}{q_{i}(z)}(i=1,2)$ is starlike in $U$. Suppose that $q_{1}(z)$ satisfies $(3.15)$ and $q_{2}(z)$ satisfies (3.2). Let $f \in \mathcal{A}$ and suppose that $\left[\frac{L(a+1, c) f(z)}{z}\right]^{\mu} \in H[q(0), 1] \cap Q$. If $\Lambda(z)$ given by (3.8) is univalent in $U$, and

$\chi+\zeta q_{1}(z)+\delta\left[q_{1}(z)\right]^{2}+\gamma \frac{z q_{1}^{\prime}(z)}{q_{1}(z)} \prec \Lambda(z) \prec \chi+\zeta q_{2}(z)+\delta\left[q_{2}(z)\right]^{2}+\gamma \frac{z q_{2}^{\prime}(z)}{q_{2}(z)}$, 
then

$$
q_{1}(z) \prec\left[\frac{L(a+1, c) f(z)}{z}\right]^{\mu} \prec q_{2}(z),
$$

and $q_{1}$ and $q_{2}$ are, respectively, the best subordinant and the best dominant of (3.21).

\section{References}

[1] R. M. Ali, V. Ravichandran, M. H. Khan and K. G. Subramanian, Differential Sandwich theorems for certain analytic functions, Far East J. Math. Sci. 15(2004), 87-94.

[2] M. K. Aouf, F. M. Al-Oboudi and M. M. Haidan, On some results for $\lambda$-spirallike and $\lambda$-Robertson functions of complex order, Publ. Instit. Math. Belgrade, 77(2005), no. 91, 93-98.

[3] T. Bulboaca, Classes of frist order differential superordinations, Demonstratio Math. 35(2002), no.2, 287-292.

[4] T. Bulboaca, A class of superordination-preserving integral operators, Indeg. Math. (N.S.) 13(2002), no.3, 301-311.

[5] T. Bulboaca, Differential Subordinations and Superordinations, Recent Results, House of Scientific Book Publ., Cluj-Napoca, 2005.

[6] B. C. Carlson and D. B. Shaffer, Starlike and prestarlike hypergeometric functions, SIAM J. Math. Anal., 15(1984), 737-745.

[7] R. M. El-Ashwah and M. K. Aouf, Differential subordination and superordination for certain subclasses of p-valent functions, Math. Comput. Modelling 51(2010), no. 5-6, 349-360.

[8] J. Dziok and H. M. Srivastava, Classes of analytic functions associated with the generalized hypergeometric function, Appl. Math. Comput., 103(1999), 1-13.

[9] S. S. Miller and P. T. Mocanu, Differential Subordinations : Theory and Applications, Series on Monographs and Texbooks in Pure and Appl. Math. No. 225 Marcel Dekker, Inc. New York, 2000.

[10] S. S. Miller and P. T. Mocanu, Subordinant of differential superordinations, Complex Variables 48 (2003), no. 10, 815-826. 
[11] M. Obradovic, M. K. Aouf and S. Owa, On some results for starlike functions of complex order, Publ. Inst. Math. (Beograd) (N.S.) 46 (60), (1989), 79-85.

[12] W. C. Royster, On the univalence of a certain integral, Michigan Math. J. 12 (1965), 385-387.

[13] H. Saitoh, A linear operator and its applications of first order differential subordinations, Math. Japon., 44(1996), 31-38.

[14] T. N. Shanmugam, V. Ravichandran and S. Owa, On sandwich results for some subclasses of analytic functions involving certain linear operator, Integral Transforms and Spec. Functions, 21(2010), no.1, 1-11.

[15] H. M. Srivastava and P. W. Karlsson, Multiple Gaussion Hypergeometric Series, Halsted Press, Ellis Horwood Limited, Chichester, John Wiley and Sons, New York, Chichester, Brisbane, Toronto, 1985.

[16] H. M. Srivastava and A. Y. Lashin, Some applications of the BriotBouquet differential subordination, J. Inequal. Pure. Appl. Math. 6 (2005), no.2, Art. 41, 1-7.

\author{
M. K. Aouf, \\ Department of Mathematics, \\ Faculty of Science, \\ Mansoura University, \\ Mansoura 35516, Egypt. \\ Email: mkaouf127@yahoo.com \\ T. M. Seoudy, \\ Department of Mathematics, \\ Faculty of Science, \\ Fayoum University, \\ Fayoum 63514, Egypt. \\ Email: tms00@fayoum.edu.eg
}

